\title{
Magnetic reversal of perpendicularly-biased
}

\section{Co/Pt multilayers}

\author{
O. Hellwig ${ }^{1}$, S. Maat ${ }^{1}$, J. B. Kortright ${ }^{2}$, and Eric E. Fullerton ${ }^{1}$ \\ ${ }^{1}$ IBM Almaden Research Center, San Jose, CA 95120 \\ ${ }^{2}$ Materials Science Division, Lawrence Berkeley National Laboratory, Berkeley, CA \\ 94720
}

We have investigated the magnetic reversal behavior of $\left[(\mathrm{Co} / \mathrm{Pt})_{4} \mathrm{Co} / \mathrm{CoO}\right]_{\mathrm{N}}$ multilayers that are exchange biased perpendicular to the film plane. We find clear differences in the nucleation properties of the reverse domains of the ascending and descending branches of the hysteresis loops. However, the evolution of the reverse domains, once nucleated, are symmetric to positive and negative field sweeps. This behavior is in contrast to many inplane biased experiments and can be understood from the collinear uniaxial magnetic anisotropy and unidirectional exchange-bias axis in our system

PACS \# 75.70.-i, 75.25.+z, 75.60.-d, 61.10.Eq 
When a ferromagnetic $(F)$ thin film in contact with an antiferromagnetic (AF) thin film is cooled through the Néel point of the AF layer in an applied magnetic field, the hysteresis loop of the F develops a loop-shift and an enhanced coercivity. ${ }^{1-4}$ These exchange biasing effects arise as the spin order of the AF is established in the presence of the F through the interfacial F-AF exchange interaction. Although exchange bias is well established, a convincing microscopic description that explains the experimentally observed phenomena has proven elusive. ${ }^{3,4}$ Recent articles review the experimental ${ }^{1,2}$ and theoretical $\mathrm{P}^{3,4}$ efforts to understand this phenomenon. Research has recently focused on the role of exchange bias on the reversal behavior of F/AF bilayers. ${ }^{5-9}$ In particular, it has been observed that in addition to shifting the hysteresis loop and enhancing the coercivity, exchange bias can also lead to asymmetrical loop shapes. This behavior has recently been related to differences in the magnetic reversal mode during the ascending and descending branches of the hysteresis loop. ${ }^{5-9}$ Asymmetric reversal appears to be a rather general property and may prove crucial to understanding many of the physical phenomena observed in exchange-biased systems.

Most studies have focused on F/AF samples where the magnetization of the ferromagnetic layer is confined to the film plane. Maat et al., recently showed exchange bias also can be observed for the magnetization perpendicular to the film plane in $\mathrm{Co} / \mathrm{Pt}$ multilayers biased by $\mathrm{CoO} .{ }^{10}$ Perpendicular bias effects have also been reported for $\mathrm{FeF}_{2} / \mathrm{CoPt}$ bilayers. ${ }^{11}$ In contrast to many in-plane biased samples, the hysteresis loops of the perpendicular biased $\mathrm{Co} / \mathrm{Pt}$ multilayers appeared symmetric about the exchange field. In this paper we use magnetic $x$-ray small-angle scattering (SAS) techniques ${ }^{12}$ to probe the symmetry of the magnetic reversal of perpendicularly-biased $\mathrm{Co} / \mathrm{Pt}$ multilayers. We 
find that there are differences in the nucleation properties of the reverse domains of the ascending and descending branches of the loop. However, the evolution of the reverse domains, once nucleated, are symmetric to positive and negative field sweeps. This behavior is in contrast to many in-plane biased experiments and is understood from the collinear anisotropy and exchange-bias axis present in our samples.

For these studies we grew $\mathrm{CoO} /(\mathrm{Co} / \mathrm{Pt})_{\mathrm{x}}$ mulitlayers on $\mathrm{S}_{3} \mathrm{~N}_{\mathrm{x}}$ coated $\mathrm{Si}$ substrates and $\mathrm{Si}_{3} \mathrm{~N}_{\mathrm{x}}$ membranes to facilitate transmission $\mathrm{x}$-ray measurements. The multilayer structure used was $\operatorname{CoO}(10 \AA)\left\{[\operatorname{Co}(4 \AA) \operatorname{Pt}(7 \AA)]_{4} \operatorname{Co}(6 \AA) \operatorname{CoO}(10 \AA)\right\}_{10}$ as shown schematically in Fig. 1. The samples were grown at room temperature by DC magnetron sputtering in a 3-mTorr Ar atmosphere onto a 200- $\AA$ Pt seed layer and capped with a 20$\AA$ Pt layer. The CoO layers were formed by oxidizing 12 - $\AA$ Co layers in ambient atmosphere. This results in a $\operatorname{Co}(\sim 6 \AA) \operatorname{CoO}(\sim 10 \AA)$ bilayer after oxidation. ${ }^{10}$ The $[\mathrm{Co}(4 \AA) / \mathrm{Pt}(7 \AA)]_{4} / \mathrm{Co} / \mathrm{CoO}$ multilayer unit cell is similar to that used to demonstrate perpendicular bias in Ref. 10 and was designed to have perpendicular anisotropy. The Xray reflectivity spectrum (Fig.1) confirms that a well-defined multilayer structure is formed. Multilayer diffractions peaks are observed from the $60-\AA[\operatorname{Co}(4 \AA) / \operatorname{Pt}(7 \AA)]_{4}$ $\operatorname{Co}(6 \AA) / \operatorname{CoO}(10 \AA)$ unit cell as well as from the $11-\AA \operatorname{Co}(4 \AA) / \operatorname{Pt}(7 \AA)$ bilayers. X-ray diffraction showed that the Pt buffer layer and the Co/Pt multilayers are (111) textured with a mosaic spread of $\sim 10^{\circ}$. The $\mathrm{Co} / \mathrm{Pt}$ multilayer diffraction displays additional superstructure reflections resulting from the $60-\AA[\mathrm{Co} / \mathrm{Pt}]_{4} \mathrm{Co} / \mathrm{CoO}$ unit cell periodicity indicating that some degree of crystal coherence is maintained through the $\mathrm{CoO}$ layers. The macroscopic magnetic properties were characterized by SQUID and Kerr magnetometry. Shown in Fig. 2 are the hysteresis loops measured at room temperature 
and at $150 \mathrm{~K}$ and $50 \mathrm{~K}$ after field cooling in a positive field sufficient to saturate the sample perpendicular to the film plane. At room temperature, the $\mathrm{CoO}$ layers are nonmagnetic and the hysteresis loop is nearly identical to that observed for a similarly grown $[\mathrm{Co}(4 \AA) / \operatorname{Pt}(7 \AA)]_{50}$ superlattice without the $\mathrm{CoO}$ layers. ${ }^{12}$ The hysteresis loop is typical of magnetic thin films with perpendicular anisotropy and is characterized by nucleation and domain wall motion. ${ }^{13}$ The fact that the room-temperature loop mimics that in multilayers without the $\mathrm{CoO}$ layers suggests that the $[\mathrm{Co} / \mathrm{Pt}]_{4}$ sublayers respond collectively where the domains in vertically adjacent $[\mathrm{Co} / \mathrm{Pt}]_{4}$ regions align via magnetostatic energies. ${ }^{14-16}$ Magnetic Force Microscope (MFM) imaging of the roomtemperature remanent magnetic state (inset Fig. 2) supports this conclusion. Stripe domains are observed that display similar widths $(\sim 120 \mathrm{~nm})$ and patterns to those observed without the $\mathrm{CoO}$ layers. ${ }^{12}$ Thus, the addition of the $\mathrm{CoO}$ layers does not significantly change the magnetic properties of the multilayers at room-temperature.

For the field-cooled loops we observe an enhanced coercivity and a loop shift towards negative field with decreasing temperature. Even though the coercivity has increased from 50 Oe at room temperature to $2.3 \mathrm{kOe}$ at $50 \mathrm{~K}$ with a $1.1 \mathrm{kOe}$ loop shift, the characteristic shape of the hysteresis loop is not significantly altered. The $[\mathrm{Co} / \mathrm{Pt}]_{50}$ multilayer at $50 \mathrm{~K}$ has a coercive field of $150 \mathrm{Oe}$, indicating that the majority of the coercivity enhancement arises from the interaction with the $\mathrm{CoO}$ layers. By monitoring the bias field vs. temperature we determine a blocking temperature $\mathrm{T}_{\mathrm{B}} \sim 200 \mathrm{~K}$, in agreement with Ref. 10 .

To probe the microscopic reversal behavior of the biased samples we use magnetic x-ray small-angle scattering (SAS). ${ }^{12}$ The X-ray scattering experiments were 
performed at the Advanced Light Source on linearly and elliptically polarizing undulator beamlines 8.0 and 4.0 , respectively. All measurements described here were made using linear polarization. We used the symmetric transmission scattering geometry described in Ref. 12 to constrain the scattering vector $\mathbf{q}=\mathbf{k}_{\mathbf{0}}-\mathbf{k}_{\mathbf{f}}$ in the film plane and to optimize coupling to the in-plane structure of the magnetic domains. The photon energy was tuned to the $\mathrm{Co} L_{3}$ resonant peak $(778 \mathrm{eV})$ to maximize the magnetic scattering contrast. SAS intensity scans were measured both as a function of $\mathrm{q}$ for fixed magnetic field $\mathrm{H}$ and hysteresis scans as a function of $\mathrm{H}$ for fixed q. Because magnetic SAS originates from deviations from uniform magnetization, and its maximum intensity is orders of magnitude stronger than the scattering at saturation, it is extremely sensitive to the nucleation process at the onset of magnetization reversal, as seen below.

Shown in Fig. 3 are SAS hysteresis scans measured at a fixed $\mathrm{q}=0.027 \mathrm{~nm}^{-1}$ of a sample similar to that shown in Fig. 2 grown on a SiN membrane. This corresponds to an in-plane length scale $2 \pi / \mathrm{q}=235 \mathrm{~nm}$ and matches the expected in-plane periodicity of the domains. The sample was field cooled in a positive field and measured at $\mathrm{T}=85 \mathrm{~K}$. Scans are shown for the first, second and twentieth field cycling. At large H, the film is uniformly magnetized and there is no magnetic contribution to the SAS signal. With the onset of reverse domains at $\mathrm{H}_{\mathrm{N}}$ the scattering increases, peaks at a field that corresponds roughly to the coercivity of the sample and then decreases to the saturation value at $\mathrm{H}_{\mathrm{S}}$ with increasing reverse field. The maximum scattering is $\sim 300$ times the signal at saturation demonstrating the sensitivity is this technique to the domain structure during reversal. Similar scattering is observed in the reverse direction; however, the shape of the SAS loop at the onset of the magnetization reversal at $\mathrm{H}_{\mathrm{N}}$ is quite different for the 
ascending branch of the hysteresis cycle. The peaks in the scattering are not located symmetrically about $\mathrm{H}=0 \mathrm{Oe}$, but are shifted to negative fields reflecting the exchange bias of the sample. Both the shift of the scattering peak and the asymmetric nucleation process disappear above $\mathrm{T}_{\mathrm{B}}$.

We see two effects with cycling of the field. The first is a systematic shift of $\mathrm{H}_{\mathrm{N}}$ and $\mathrm{H}_{\mathrm{S}}$ towards the origin. The second is an increase in the peak intensities with cycling. Both effects are more pronounced for the descending than the ascending branch of the hysteresis cycle and can be interpreted as training effects that are often observed in exchange-biased structures. Training most commonly appears in polycrystalline AF layers. It is often characterized by a decrease in the measured exchange bias and coercive field with field cycling. ${ }^{1}$ and is linked to relaxation of domains in the AF layer that are field cooled into metastable configurations. The training observed here is most dramatic comparing the first and second cycle and then shows increasingly smaller difference with each field cycling. However, we are able to still resolve changes in the peak intensity up to the twentieth cycle.

Scans of $\mathrm{H}$ and $\mathrm{q}$ after 20 cycles reveal that the domain structure mediating reversal is essentially symmetric with respect to direction, although the nucleation asymmetry persists. Shown in Fig. $4 \mathrm{a}$ is a comparison of the ascending and descending branch of the SAS loop, with the field values corrected for the average bias value and then plotted vs. the absolute field value. The descending and ascending branch are represented by solid and open symbols, respectively. We find that, while there are differences in the initial nucleation processes, once nucleated the reversal behavior of the sample after training appears identical in both the field-dependent shape of the SAS loop 
as well as the absolute intensities. For the initial field cycle, we observe a $\sim 4 \%$ difference in the peak intensities which decreases to $<1 \%$ by the tenth cycle.

Shown in Fig. 4b are q-scans obtained at field values (-2.8 and 2.1 kOe) that roughly correspond to the peak intensities in Fig. 4a. The lack of a strong peak in these $q$ scans is consistent with a relatively disordered domain structure during reversal. Because the q scans measure the spatial frequency of this domain structure, their near equivalence on ascending and descending branches confirms that the domain distribution is nearly equivalent on reversal in both directions in the biased state. We note that the q scan (not shown) for this sample when demagnetized (either biased at low $\mathrm{T}$ or at room $\mathrm{T}$ ) shows a clear peak similar to those in ref. 12 , indicating that exchange bias alters the domain distribution during reversal.

Together the symmetric $\mathrm{q}$ and $\mathrm{H}$ scans reveal that the ensemble average domain structure that characterizes the reversal process is symmetric to the field sweep direction. The specific local domain structure, however, could differ with field cycling and would require magnetic imaging ${ }^{9,17}$ or coherent scattering ${ }^{18}$ to determine the specific microscopic reversal pathway. These results are in contrast to those observed in Ref. 6, for example, where dramatic differences between ascending and descending branches are observed in magnetization, polarized neutron reflectivity, and magnetoresistance measurements. There it was concluded that the descending branch of the hysteresis loop switched via coherent rotation while the ascending branch reversed by domain-wall nucleation and propagation. This particular difference in the reversal modes was related to the twinned microstructure of the AF layer. Asymmetric reversal observed in Refs. 8 and 9 were for polycrystalline $\mathrm{F}$ and AF layers with in-plane bias where the main 
differences observed in the reversal mechanism was dramatic asymmetries in the initial nucleation process with ascending and descending branches of the loop.

Our results suggest that asymmetric reversal is not an inherent property of exchange-biased systems but depends on the anisotropy and microstructure of the constituent layers. For the present samples the ferromagnetic layers have strong uniaxial anisotropy that limits potential reversal pathways. The bias then acts as an effective field that shifts the loop but does not fundamentally alter the reversal behavior. That we observe a difference in the nucleation process agrees with the discussions of the origin of asymmetric loops in Refs. 8 and 9. This difference in the nucleation suggests local regions that are more strongly exchange biased than the average. Local variations in the exchange bias have been postulated by random field theory ${ }^{19}$ and have also been experimentally observed in magnetic imaging of exchange-bias bilayers. ${ }^{9,} 17$ If present, the stronger biased regions would only effect the nucleation on the ascending branch of the hysteresis loop. For the present samples, these initially nucleated domains in the ascending branch are not sufficient to initiate a complete reversal and remain pinned. Instead reversal only occurs at higher applied fields and is controlled by the uniaxial magnetic anisotropy, dipolar energies, and domain wall pinning in the multilayer with the exchange bias acting as an effective field. However, this may not be a general property of perpendicular biased samples. For samples with stronger biasing compared to the anisotropy or dipolar energies, asymmetric reversal may be expected to reemerge.

In conclusion, we have investigated the magnetic reversal behavior of $\mathrm{Co} / \mathrm{Pt}$ multilayers under perpendicular exchange bias. We find that there are clear differences in the nucleation properties of the reverse domains of the ascending and descending 
branches of the loops in agreement with Refs. 8 and 9. However, the evolution of the reverse domains, once nucleated, are symmetric to positive and negative field sweeps for samples studied. This behavior is in contrast to many in-plane biased experiments and may be understood from the collinear uniaxial magnetic anisotropy and unidirectional exchange-bias axis in the system. This work also suggests in-plane biased systems with uniaxial in-plane magnetic anisotropy may also appear more symmetric in their reversal behavior.

We would like to thank M. Best for her help with the MFM imaging. Work at LBNL was supported by the Director, Office of Energy Research, Office of Basic Energy Sciences, MSD, of the U.S. DOE under Contract No. DE-AC03-76SF00098. O.H. was partially supported by the Deutsche Forschungsgemeinschaft via a Forschungsstipendium under contract number HE 3286/2-1. 


\section{References}

1. J. Nogués and I. K. Schuller, J. Magn. Magn. Mat. 192, 203 (1999).

2. A. E. Berkowitz and K. Takano, J. Magn. Magn. Mat. 200, 552 (1999).

3. R. L. Stamps, J. Phys. D: Appl. Phys. 33, R247 (2000).

4. M. Kiwi, J. Magn. Magn. Mater. 234, 584 (2001).

5. M. R. Fitzsimmons, P. C. Yashar, C. Leighton, J. Nogués, J. Dura, C. F. Majkrzak, and I. K. Schuller, Phys. Rev. Lett. 84, 3986 (2000).

6. C. Leighton, M. R. Fitzsimmons, P. Yashar, A. Hoffman, J. Nogués, J. Dura, C. F. Majkrzak, and I. K. Schuller, Phys. Rev. Lett. 86, 4394 (2001).

7. C. Leighton and I. K. Schuller, Phys. Rev. B 63, 174419 (2001).

8. V. I. Nikitenko, V. S. Gornakov, A. J. Shapiro, R. D. Shull, K. Liu, S. M. Zhou, and C. L. Chien, Phys. Rev. Lett. 84, 765 (2000).

9. H. D. Chopra, D. X. Yang, P. J. Chen, H. J. Brown, L. J. Swartzendruber, and W. F. Egelhoff, Phys. Rev. B 61, 15312 (2000).

10. S. Maat, K. Takano, S. S. P. Parkin, Eric E. Fullerton, Phys. Rev. Lett. 87, 087202 (2001).

11. K. Kagerer, Ch. Binek, W. Kleemann, J. Magn. Magn. Mater. 217, 139 (2000).

12. J. B. Kortright, S.-K. Kim, G. P. Denbeaux, G. Zeltzer, K. Takano, and Eric E. Fullerton, Phys. Rev. B 64, 092401 (2001).

13. C. Kooy and U. Enz, Philips Res. Repts. 15, 7 (1960).

14. H. J. G. Draaisma and W. J. M de Jonge, J. Appl. Phys. 62, 3318 (1987).

15. S. Honda, Y. Ikegawa, and T. Kusuda, J. Magn. Magn. Mater. 111, 273 (1992).

16. S.-B. Choe and S.-C Shin, Phys. Rev. B 59, 142 (1999). 
17. F. Nolting, A. Scholl, J. Stöhr, J. W. Seo, J. Fompeyrine, H. Siegwart, J.-P. Locquet, S. Anders, J. Lüning, E. E. Fullerton, M. F. Toney, M. R. Scheinfein, and H. A. Padmore, Nature 405, 767 (2000).

18. B. Hu, P. Geissbuhler, L. Sorensen, S. D. Kevan, J. B. Kortright, E E. Fullerton, Synch. Rad. News 14, 11 (2001).

19. A.P. Malozemoff, Phys. Rev. B 35, 3679 (1987); J. Appl. Phys. 63, 3874 (1988); Phys. Rev. B 37m 7673 (1988). 


\section{Figure Captions:}

Figure 1: Low-angle x-ray reflectivity of a $\left[(\operatorname{Co}(4 \AA) / \operatorname{Pt}(7 \AA))_{4} \operatorname{Co}(6 \AA) / \operatorname{CoO}(10 \AA)\right]_{10}$ superlattice structure. The structure is shown schematically in the inset. The periodicity of both the $\mathrm{CoO}$ layers and the $\mathrm{Co} / \mathrm{Pt}$ multilayers are reflected in the scan.

Figure 2: Out-of-plane hysteresis loops for a $\left[(\operatorname{Co}(4 \AA) / \operatorname{Pt}(7 \AA))_{4} / \operatorname{Co}(6 \AA) / \operatorname{CoO}(10 \AA)\right]_{10}$ superlattice structure measured at room temperature $(300 \mathrm{~K})$ and at 50 and $150 \mathrm{~K}$ after field cooling in a positive magnetic field. The nucleation field $\mathrm{H}_{\mathrm{N}}$ and saturation field $\mathrm{H}_{\mathrm{S}}$ for the descending branch of each hysteresis loop are indicated. The inset shows the $5 \times 5$ $\mu \mathrm{m}^{2}$ MFM image of the room-temperature domain pattern at remanance. The dark and light contrast corresponds to the positive and negative out-of-plane magnetization, respectively.

Figure 3: (a) Field-dependent small-angle scattering measurements of a perpendicular $\left[(\operatorname{Co}(4 \AA) / \operatorname{Pt}(7 \AA))_{4} \operatorname{Co}(6 \AA) / \operatorname{CoO}(10 \AA)\right]_{10}$ superlattice structure. The sample was positive field cooled to $85 \mathrm{~K}$ where the first, second and twentieth field cycle is shown. $\mathrm{H}_{\mathrm{N}}$ and $\mathrm{H}_{\mathrm{S}}$ indicate the nucleation and saturation fields, respectively, for the descending branch of the hysteresis loop. The arrows indicate the field-sweep directions.

Figure 4: Field-dependent small-angle scattering measurement of the perpendicular $\left[(\operatorname{Co}(4 \AA) / \operatorname{Pt}(7 \AA))_{4} \operatorname{Co}(6 \AA) / \operatorname{CoO}(10 \AA)\right]_{10}$ superlattice structure after field cycling 20 times. The data is corrected for the average bias field $\mathrm{H}_{\mathrm{E}}$ and then plotted vs. absolute field to compare the SAS for decreasing (filled circles) and increasing (open circles) field sweeps. (b) q-scans measured in an applied field of $\mathrm{H}=-2.8 \mathrm{kOe}$ after positive saturation (descending branch) and $\mathrm{H}=2.1 \mathrm{kOe}$ after negative saturation (ascending branch). 


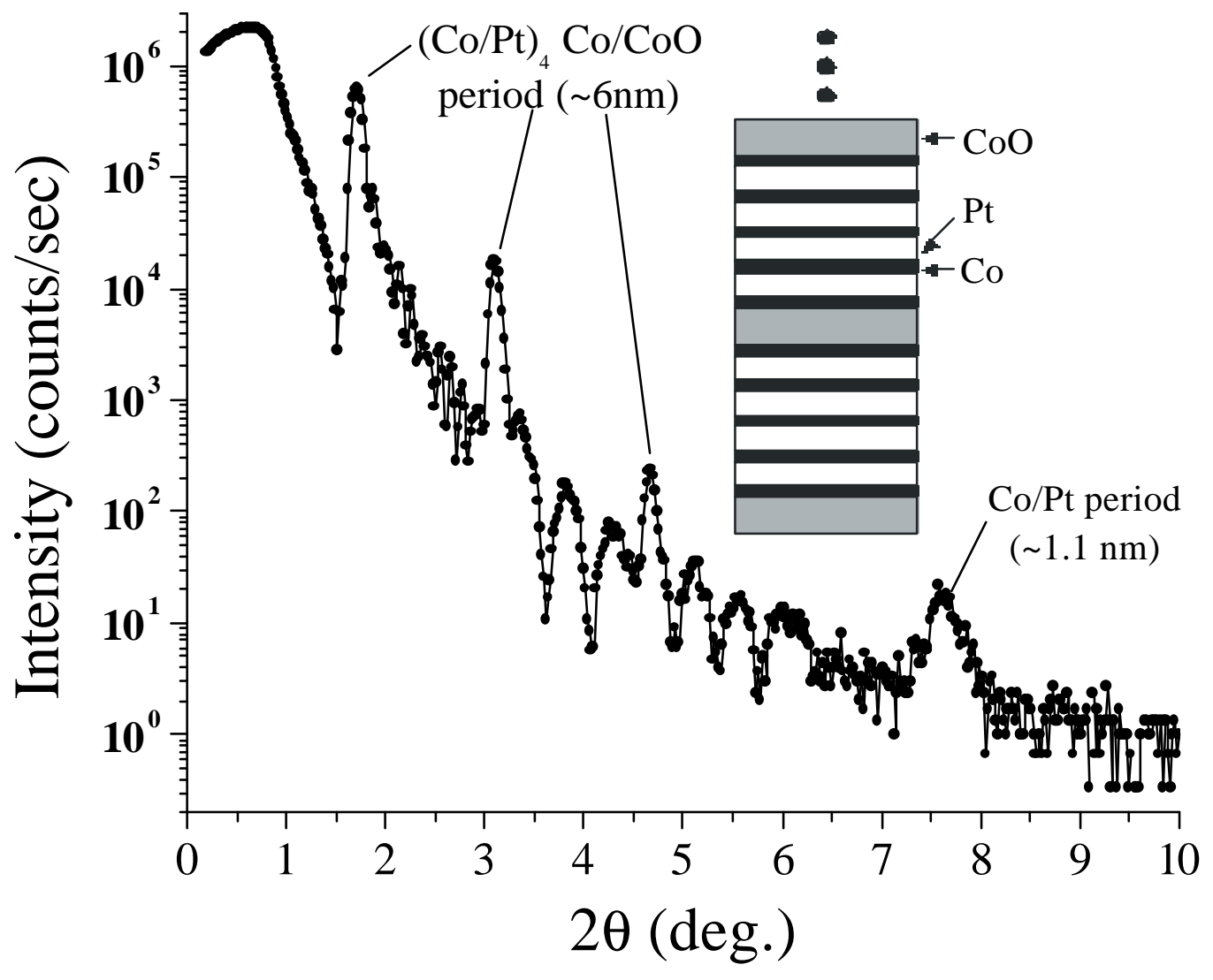

Figure 1, Hellwig et al. 


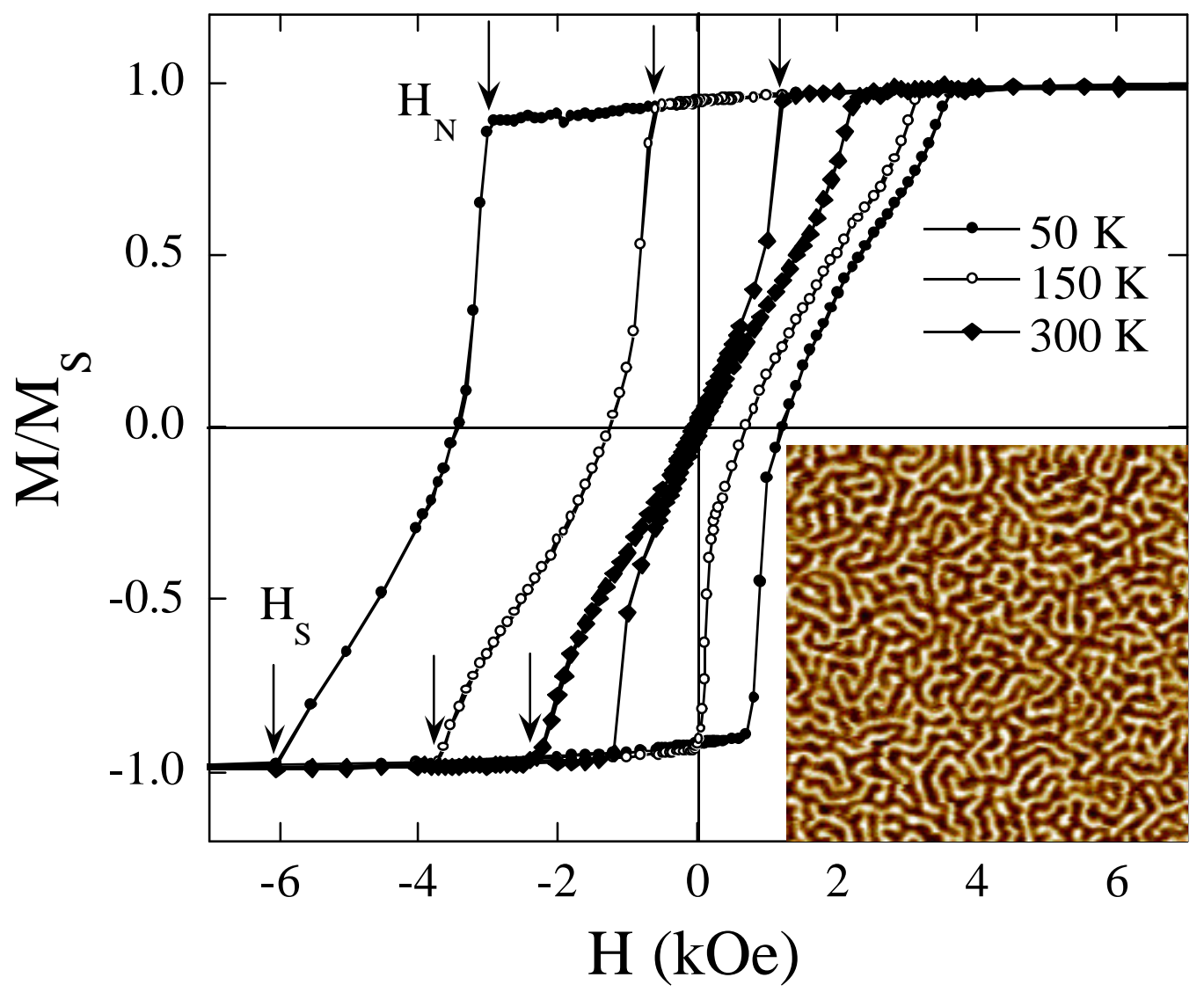

Figure 2, Hellwig et al. 


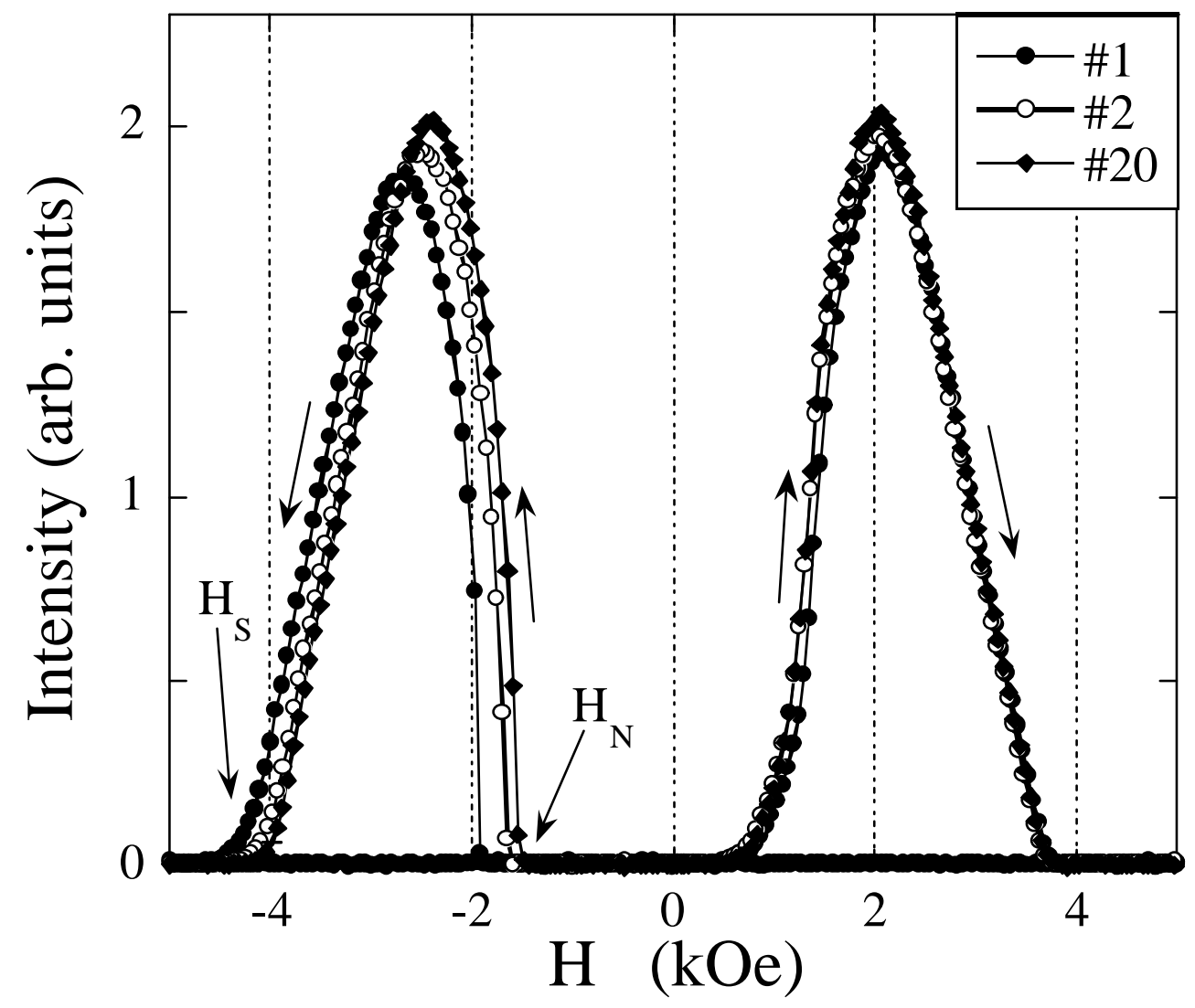

Figure 3, Hellwig et al. 

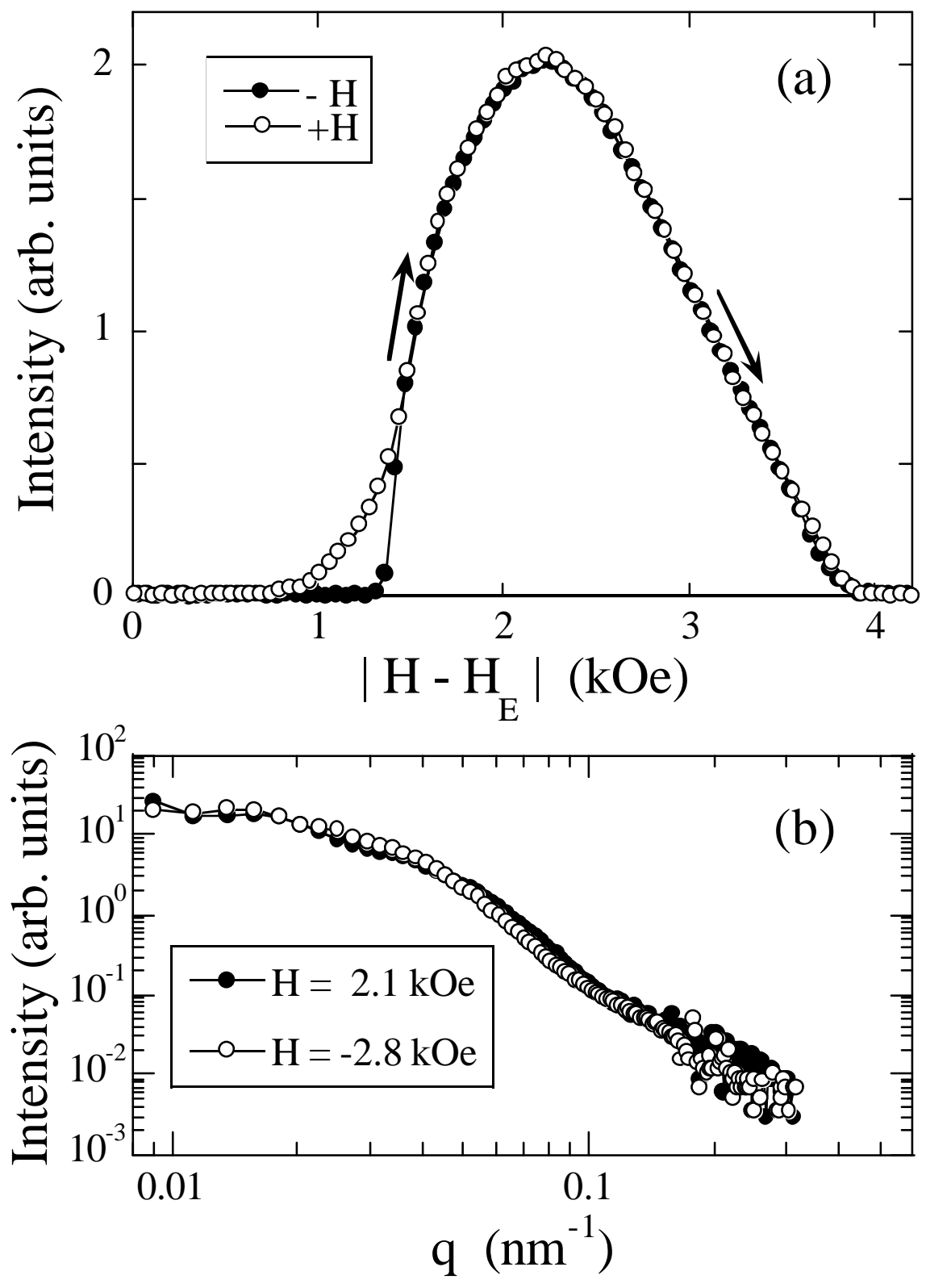

Figure 4, Hellwig et al. 